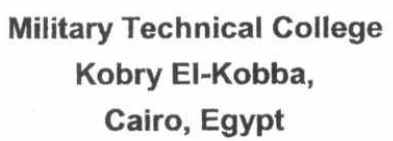

11-th International Conference

on Aerospace Sciences \&

Aviation Technology

\title{
DESIGN, ANALYSIS, AND RELIABILTY OF SOLAR PANEL ROTATION MECHANISM FOR MICROSATELLITE
}

\author{
Gasser F. Abdelal ${ }^{1}$, Mohamed Kassab²
}

\begin{abstract}
Solar array rotation mechanism provides a hinged joint between the solar panel and satellite body, smooth rotation of the solar array into deployed position and its fixation in this position. After unlocking of solar panel (while in orbit), rotation bracket turns towards ready-to-work position under the action of driving spring. During deployment, once reached the required operating angle (defined by power subsystem engineer), the rotation bracket collides with the fixed bracket that is mounted on body of the satellite, to stop rotation. Due to the effect of collision force that may alter the rotation mechanism function, design of centrifugal brake is essential. At stoppage moment micro-switches activate final position sensor and a stopper locks the rotation bracket. Design of spring and centrifugal brake components, static finite element stress analysis of primary structure body of rotation mechanism at stoppage moment have been obtained. Last, reliability analysis of rotation mechanism is evaluated. The benefit of this study is to aid in the design of rotation mechanism that can be used in micro-satellite applications.
\end{abstract}

\section{Keywords}

Aerospace, Satellite, Structure, Rotation Mechanism, Spring, Centrifugal Brake, Finite Element, and reliability

\footnotetext{
${ }^{1}$ Gasser F. Abdelal, Ph.D., Deputy Group Leader - Satellite Structure, National Authority for Remote Sensing and Space Science (NARSS).

${ }^{2}$ Mohamed Kassab, Senior Structure Engineer - Satellite Structure, National Authority for Remote Sensing and Space Science (NARSS).
} 


\section{Nomenclature}

$\mathrm{J}$ the moment of inertia of a rotation element (the solar panel array) concerning an axis of rotation; $\mathrm{kg} \cdot \mathrm{m}^{2}$

$\mathrm{J}_{\mathrm{r}} \quad$ the moment of inertia of rotating parts of the mechanism; $\mathrm{kg} \cdot \mathrm{m}^{2}$

$\Phi \quad$ the current rotation angle counted from initial position of a rotation element; (rad.)

$M_{\text {res }}$ the total moment of forces of resistance; Kgf.mm

$M_{c} \quad$ the moment of resistance to bend a cable; Kgf.mm

$\lambda$ factor taking into account rigidity of a cable at various temperatures (it is defined experimentally).

$\mathrm{M}_{\mathrm{f} . \mathrm{c} . \mathrm{b}}$ the resulted moment of friction due to resist the rotation part of centrifugal brake with internal surface of the cartridge; Kgf.mm

$\mathrm{M}_{\mathrm{f}}$ the moment of friction in centrifugal brake multiplication ratio of gears in centrifugal brake; Kgf.mm

C rigidity of a spring.

$M_{m . s}$ the moment needed to overcome of micro switch resistance.

$F \quad$ the force necessary for operation of the micro switches; Kgf.mm

$\mathrm{K}_{0} \quad$ factor curvature.

$M_{1} \quad$ the working moment of a spring corresponding to the final position of solar panels; Kgf.mm

$M_{2}$ the working moment of a spring corresponding to the initial position of solar panels; Kgf.mm

$\Phi_{1} \quad$ the working compression angle of a spring corresponding to the final position of solar panels; degree.

$\Phi_{2} \quad$ the working compression angle of a spring corresponding to the initial position of solar panels; degree.

$\varphi^{\prime \prime} \quad$ angular acceleration; $r / \mathrm{s}^{2}$

$\alpha \quad$ angle of an inclination of coils of the spring; degree

$\omega \quad$ Angular velocity at the end of accelerating of the panel; $r / s e c$

$t_{\text {acc }} \quad$ time of accelerating of solar panel, sec

$\mathrm{V}$ linear velocity of rotation of the center of gravity of panel; $\mathrm{m} / \mathrm{s}^{2}$

$\mathrm{R} \quad$ radius of rotation of the center of gravity of panel; $\mathrm{mm}$

$\mathrm{N}$ normal reaction of the cartridge working; $\mathrm{kgf}$

a shoulder of action of force $\mathrm{N}$ concerning an axis of rotation $\mathrm{B} ; \mathrm{mm}$

$\mathrm{F}_{f} \quad$ friction force; $\mathrm{kgf}$

d shoulder of action of force $F_{f}$ concerning an axis of rotation $B ; m m$

$\mathrm{P}_{\mathrm{c}} \quad$ centrifugal force; $\mathrm{kgf}$

b shoulder of action of force $P_{c}$ concerning an axis of rotation; $m$

$M_{\text {spr }}$ the moment of a centrifugal brake spring; $\mathrm{kgf} . \mathrm{mm}$

$\rho$ distance from an axis of rotation to the center of gravity; $\mathrm{mm}$

$\mathrm{G}$ weight of rotation part of centrifugal brake; Kgf

$\omega_{0} \quad$ angular velocity of rotation of axis (B) about the center of rotation (O); r/sec

$f$ factor of friction between pair of rotation parts and the inner surface of the case (steel - bronze), $f=0.18 ;[1]$. 


\section{Introduction}

Rotation mechanism assembly consists from three subassemblies. Each subassembly consists from three to four parts. Fig. 1 shows a general view of microsatellite while solar panels are deployed using rotation mechanism. Technical specification and structural design of the rotation mechanism are presented in Fig. 2 .

Subassembly ' $A$ ' as shown in Fig.4 consists from; fixed part that is mounted to the body of the Satellite, bracket that its function is to stop the rotating part of mechanism, which is connected to solar panel, Gear Housing that its function is to house the gears and centrifugal brake, and flange that its function is a cover for the gear housing and fixes it on the axis of rotation. Subassembly 'B' as shown in Fig. 5 consists from; Rotating part that is connected to the solar panel, Bracket that its function is to fix the internal gear to the rotation axis of the rotating part, and Bolt that its function is to transfer rotation from internal gear to the rotation part. Subassembly ' $\mathrm{C}$ ' as shown in Fig. 6 consists from; Internal Gear that its function is to transfer rotation from gears to the rotating part and Bracket-2 that works with Bracket-1 from subassembly ' $A$ ' to stop the rotating part at the designed deployed angle of solar panel. Subassembly ' $A$ ' is fixed to satellite body and has the rotation gears, while subassembly ' $B$ ' is fixed to solar panel. Subassembly ' $C$ ' transfers rotation motion between subassemblies ' $A$ ' and ' $B$ '.

Six-stage multiplier and centrifugal brake are built in the fixed part. Final position sensor and stopper are mounted on bracket for mounting of rotation mechanism. Pushers of the sensor micro switches are installed on rotation bracket.

Solar array is mounted to the rotation bracket using two screws M4. In initial (stowed) position installation surfaces of flange of the satellite body and installation surfaces of flange of the rotation mechanism are in the same plane. Rotation bracket with solar array is held in this position by locking mechanisms.

Rotation bracket together with solar array turns towards ready-to-work position under the action of driving spring after unlocking. Gear of the drive shaft puts the centrifugal brake in action. Mass of the rotation mechanism is $0.22 \mathrm{~kg}$, as recommended by satellite configuration engineer.

Rotation mechanisms of mechanical (spring) type work with the use of a various kind of elastic energy sources with special centrifugal brake devices to reduce the rotation velocity of a rotation element.

Once Micro-Satellite is in orbit, the solar panels are deployed to resume their function. Solar panels are deployed through Rotation Mechanism with acceleration $\alpha(t)$. Results from solar panel simulation (forces and moments at fixation nodes) are applied here as loads. 


\section{Description of motion}

The rotation element, for example the solar panel array, by means of the gear multiplier is connected to a centrifugal brake.

At achievement of angular speed $\omega_{c}$ the centrifugal brake creates the moment of friction, which slows down rotation of a rotation element.

The differential equation of a rotation element movement around an axis of rotation of the spring mechanism with a centrifugal brake is expressed by the next formula:

$$
\left(J+J_{r}\right) \ddot{\varphi}=\mathrm{M}-\sum \mathrm{M}_{\mathrm{res}}
$$

$M$ - the working moment of a torsion spring, $(M=f(\Phi))$. The total moment of resistance $\left(M_{\text {res }}=M_{c}+M_{f . c . b}\right)$. $M_{c}$ depends on temperature of the cable that transfers power from solar panel to the power subsystem. It is necessary to expect for possible extreme values of that moment. It is possible to present the following expression; $\left(M_{c}=\lambda M_{0}\right)$, Where, $M_{0}$ is the value of the initial moment developed by spring drive: At maximum value; $M_{c \max }=\lambda_{\max } M_{0}$; (should satisfy condition, $\lambda_{\max }<1$ ).

$$
\begin{aligned}
& M_{f, c . b}=f\left(\varphi, \mathrm{f}_{\mathrm{f}}\right) \\
& \mathrm{M}_{\mathrm{f} . \mathrm{c} . \mathrm{b}}=\mathrm{M}_{\mathrm{f} \cdot i^{2}}
\end{aligned}
$$

Thus, the basic components of the total moment of resistance are the moment of resistance to bend a cable during rotation and the resulted moment of friction due to centrifugal brake. Thus, for any considered type of a drive the generalized differential equation of movement of a rotation element becomes:

$$
\left(J+J_{r}\right) \ddot{\varphi}=\mathbf{M}-\lambda \mathbf{M}_{0}-\mathbf{M}_{f . c . b}
$$

Then the equation (2.1)can be rewritten down as follows:

$$
J_{\Sigma} \ddot{\varphi}=c \varphi-\lambda M_{0}-M_{\mathrm{f}} i^{2}
$$

The solution of this differential equation is a complex mathematical equation; therefore we use a traditional technique of engineering calculation with four basic steps:

1. Determine the general initial geometrical and physical parameters;

2. Definition of the necessary gear multiplier, which provides the suitable operating rotation time;

3. Definition of a design of the torsion spring of the rotation mechanism; 4. Definition of a design of the brake device and calculate the opening time of
the mechanism. 


\section{INITIAL DATA}

-The moment of inertia of solar array panel relative to the rotation mechanism axes (Calculated by Satellite configuration engineer) $=0.017(0.167)$ kgf.m.sec2 (kg.m2) -Solar array panel rotation angle (Requirements given by satellite system engineer) $=90 \mathrm{deg}$.

-Time of rotation of solar array panel not less than (Requirements given by satellite structure and mechanisms engineer) $=1.5 \mathrm{sec}$.

-External diameter of the rotation mechanism case (Requirements given by satellite structure and mechanisms engineer) $=35 \mathrm{~mm}$.

-Weight of the mechanism not more than (Requirements given by satellite configuration engineer) $=0.22 \mathrm{Kgf}$.

\section{SPRING DESIGN}

We determine the moment of forces of resistance, which the spring should overcome at the end of rotation $\left(M_{R}\right)$ :

$$
\begin{aligned}
& \mathrm{M}_{\mathrm{R}}=2 \mathrm{M}_{\mathrm{m} \cdot \mathrm{s}}+\mathrm{M}_{\mathrm{c}} \\
& \mathrm{M}_{\mathrm{m} \cdot \mathrm{s}}=F L
\end{aligned}
$$

F ranges from 0.1:0.23 kgf (given by micro-switch supplier); for calculation we choose maximum force $F=0.23 \mathrm{kgf}$.

The normal distance of the arm of force to the button of micro switch $(L=0.024 \mathrm{~m})$. In our mechanism 2 micro switches are used $\left(2 \mathrm{M}_{\mathrm{m} . \mathrm{s}}=0.01104 \mathrm{kgf.m}\right)$, one switch is basic and the other is redundant.

$M_{c}$ is determined experimentally by YOZHNOYE Design Office Denpropetrovsk UKRAINE, which equals $0.07 \mathrm{kgf}$.m. Taking into account possible discrepancies at carrying out of experiment, so, we enter a factor of stock $T_{0}=1.3$;

$$
\begin{aligned}
& M_{c}=(0.07)\left(T_{0}\right)=0.091 \mathrm{kgf.m} \\
& M_{R}=2 M_{m . s}+M_{c}=0.102 \mathrm{kgf.m}
\end{aligned}
$$

According to the document working on the enterprise [11], there will be a deviation due to manufacturing conditions. It is limited in $\pm 10 \%$, then $M_{R}=(0.102)(1.1)=$ 0.112 Kgf.mm.

For spring material it is chosen; a wire of steel $12 \times 18 \mathrm{H} 10 \mathrm{~T}$ [12]; $d=2.81 \mathrm{~mm} ; \mathrm{E}=$ $1.85 \times 10^{4} \mathrm{kgf} / \mathrm{mm}^{2} ; \sigma_{\mathrm{u}}=(175 \ldots 205) \mathrm{kgf} / \mathrm{mm}^{2} ; \sigma_{\text {all }}=123 \mathrm{kgf} / \mathrm{mm}^{2}$. Structurally, for the spring we assume; External diameter of the spring $D_{1}=30.97 \mathrm{~mm}$, internal diameter of the spring $D_{2}=25.35 \mathrm{~mm}$, and average diameter of the spring $D=28.16 \mathrm{~mm}$. Maximum permissible torsion moment of the spring: 


$$
\begin{aligned}
& M_{\max }=\frac{\pi \times d^{3} \times\left[\sigma_{\text {all }}\right]}{32 \times K_{\circ}}=247.369 \\
& K_{\circ}=\frac{4 j-1}{4 j-4} \\
& j=\frac{D}{d}=\frac{28.16}{2.81}=10.02258
\end{aligned}
$$

Assume $M_{1}$ equals the minimum resistance moment $M_{R}$, while $M_{3}$ equals the maximum resistance moment $M_{\max }$. Assume $M_{2}$ is less than $M_{\max }(M 2=240$ Kgf.mm). The rotation angle is specified: $\left(\varphi_{2}-\varphi_{1}=90^{\circ}\right)$. Determine rigidity of the spring:

$$
c=\frac{M_{2}-M_{1}}{j_{2}-j_{1}}=\frac{240-112}{90}=1.422 \mathrm{Kgf} \cdot \mathrm{mm} / \mathrm{deg}
$$

Angles of a twisting:

$$
\varphi_{1}=\frac{\mathrm{M}_{1}}{\mathrm{c}}=78.75^{\circ}, \quad \varphi_{2}=\frac{\mathrm{M}_{2}}{\mathrm{c}}=168.75^{\circ}, \quad \varphi_{3}=\frac{\mathrm{M}_{3}}{\mathrm{c}}=173.93^{\circ}
$$

$\varphi_{1}=79^{\circ}$ (as $78.75^{\circ}$ is immeasurable), thus; $M_{1}=\varphi_{1} . c=112.35 \mathrm{Kgf} \cdot \mathrm{mm} ; \varphi_{2}=\varphi_{1}+$ $90^{\circ}=169^{\circ} ; M_{2}=\varphi_{2} . c=240.35 \mathrm{Kgf} . \mathrm{mm}$. Rigidity of one coil of the spring $C^{\prime}$ :

$$
C^{\prime}=\frac{E \times d^{4}}{64 \times D \times(57.3)}=11.16939 \mathrm{Kgf} \cdot \mathrm{mm} / \mathrm{deg}
$$

Number of working coils of the spring $\mathrm{n}, n=C^{\prime} / C=7.85$. We specify maximum permissible twisting angle of the spring,

$$
\varphi_{3}=\frac{360 \times \mathrm{D} \times \sigma_{\mathrm{all}} \times \mathrm{n}}{\mathrm{d} \times \mathrm{E} \times \mathrm{K}_{0}}=166^{\circ}
$$

Take clearance between turns of the spring $\mathrm{f}_{3}{ }^{\prime}$ [13],

$$
\frac{f_{3}^{\prime}}{d}=0.45 \pm 0.20, \quad f_{3}^{\prime}=\left(\frac{f_{3}^{\prime}}{d}\right) d=(0.45 \pm 0.20)(2.81)
$$

Pitch of a spring, $\left(t=f_{3}+d=4.07 \mathrm{~mm}\right)$. Length of a working part of the non-loaded spring $\left(\boldsymbol{I}_{0}=\mathrm{t} . \mathrm{n}+\mathrm{d}=33.33 \mathrm{~mm}\right)$. Mean diameter ( $\left.\mathrm{D}^{\prime}\right)$ of the spring after loaded with moment $\mathrm{M}_{2}$ is, 


$$
\mathrm{D}^{\prime}=\frac{\mathrm{D}}{1+\frac{\varphi_{2(\mathrm{rad})}}{2 \pi \times \mathrm{n}}}=26.5 \mathrm{~mm}
$$

Internal diameter of a spring after loaded with moment $\mathrm{M}_{2}, D_{2}^{\prime}=D^{\prime}-d=23.69 \mathrm{~mm}$. Increase in length of the spring after loaded with moment $\mathrm{M}_{2}$ :

$$
\Delta \mathrm{l}=\varphi_{2} \mathrm{D} / 2 \operatorname{Sin} \alpha=1.9 \mathrm{~mm}, \quad \tan \alpha=\frac{\mathrm{d}+\mathrm{f}_{3}^{\prime}}{\pi \times \mathrm{D}}
$$

Length of the spring after loaded with moment $\mathrm{M}_{2}, L=l_{0}+\Delta l=35.24$. Axial length of the developed spring without taking into account hooks, $L_{a}=(3.2) D . n=675.84 \mathrm{~mm}$ Increase the length of the spring at the two ends by $5 \mathrm{~mm}$ for hooks, the total axial length of the spring, $L_{a}$ tot $=675.84+(2)(5)=685.84=686 \mathrm{~mm}$. Mass of the spring, $G=V \cdot \gamma=33.393 \mathrm{gm},\left(\gamma=7.85 * 10^{-6} \mathrm{Kgf} / \mathrm{mm}^{3}\right), V=\pi{ }^{*} r^{2}{ }^{*} L_{a \text { tot }}=4254.28 \mathrm{~mm}^{3}$.

\section{DETERMINATION OF THE MOMENT OF THE FORCE NECESSARY FOR ACCELERATING OF SOLAR PANEL AFTER RELEASING}

The moment of force necessary for accelerating of solar panel after release:

$$
\begin{aligned}
& \sum M_{\text {res }}=M_{\text {inertia }}+M_{c}+2 \cdot M_{\text {m.s }} \\
& M_{\text {interia }}=J_{\text {s.p }} \ddot{\varphi} \\
& \omega=V / R \mathrm{rad} / \mathrm{sec}
\end{aligned}
$$

Velocity of the center of gravity of the center of mass of solar panel $\left(V={ }_{n} D n\right)$, where $n$ is number of revolution per seconds, $\omega=2 \pi n \mathrm{r} / \mathrm{s}$. Summation of all resistance moments equal, $\left(\sum M_{\text {res }}=M_{\text {inertia }}+M_{c}+2 M_{m . s}=0.11817 \mathrm{Kgf} . \mathrm{m}\right)$. There will be a deviation due to manufacturing conditions that is limited in $\pm 10 \%$, so $\sum \mathrm{M}_{\text {res }}=$ $(1.1)(118.17)=129.98$ Kgf.mm.

\section{DESIGN OF SPEED REGULATOR}

The speed regulator composed of a centrifugal brake and gear multiplicator. The gear multiplicator engaged with the rotating part of the rotation mechanism by means of an epicyclical gear integrated with the rotary case. As well as the second end of the gear multiplicator is connected to the centrifugal brake. 
The principle of centrifugal braking is dissipating a part of the mechanical energy stored in the spring during rotation of the solar panel and transforms it to thermal energy due to friction between the high speed rotation part and the case surface of the brake device. This process leads to a decrease in the angular velocity of the solar panel rotation and the total time needed to complete the rotation.

The centrifugal brake is shown in fig.3. To determine $\omega_{0}$, write the moment equation around the hinge $B$ :

$$
\begin{aligned}
& N a+F_{f} d+M_{s p r}-P_{c} b=0 \\
& P_{c}=\frac{G}{g} \omega_{o}^{2} \rho, \quad N=\frac{F_{f}}{f} \\
& F_{f}=\frac{\frac{G}{g} \omega_{o}^{2} \rho b-M_{s p r}}{\frac{a}{f}+d}
\end{aligned}
$$

At the moment, $\omega_{0}=\omega_{\text {o.crit. }}$, the sum of the moments of forces of friction concerning an axis of rotation $(\mathrm{O})$ of a framework counterbalances moment $\mathrm{M}_{0}$ :

$$
2 F_{f} r=M_{0} \quad(r \text { is radius of case })
$$

Substituting value $F_{f}$, we receive:

$$
\begin{gathered}
M_{0} / 2 r=\frac{\left[\left(\frac{G}{g}\right) \cdot \omega_{0}^{2} \cdot \rho \cdot b-M_{s p r}\right]}{\left(\frac{a}{f}+d\right)} \\
\omega_{\text {o. crit. }}=\sqrt{\frac{\frac{M_{0}}{2 r}\left(\frac{a}{f}+d\right)+M_{s p r}}{G \rho b}} g \\
\omega_{\text {o. crit. }}=\sqrt{K M_{0}}, \quad K=\frac{\frac{1}{2 r}\left(\frac{a}{f}+d\right)+\frac{M_{s p r}}{M_{0}}}{G p b} g
\end{gathered}
$$

$M_{\text {spr }}$ at small speed of rotation or at case of rest, should provide contact between mass to the hub of a framework, $M_{\text {spr. }}=G$.L. Where $G$ is the mass of centrifugal brake, $=0.0049 \mathrm{kgf}$, and $\mathrm{L}$ is the distance between the center of gravity of the rotating part and its hinge. $=6.12 \mathrm{~mm} . \mathrm{M}_{\mathrm{spr} .}=0.0299 \mathrm{kgf} . \mathrm{mm}$. 
According to the document [14], there will be a deviation due to manufacturing conditions. It is limited in $\pm 10 \%$, then $M_{\text {spr. }}=(0.0299)(1.1)=0.033 \mathrm{Kgf} . \mathrm{mm}$.

We shall determine $\omega_{\text {o.crit. }}$ for two cases, at $M_{1}$ and at $M_{2}$ of the driving spring of the mechanism. There are correspondingly two of rotation moments of centrifugal brake spring,

$$
M_{10}=\frac{M_{1}}{i} \eta, \quad M_{20}=\frac{M_{2}}{i} \eta
$$

Where $i$-multiplication ratio of gears; $(i=276,5476)$.

$\eta$-multiplier efficiency,

$$
\begin{aligned}
& \eta=\eta_{1} \cdot \eta_{2} \cdot \eta_{3} \cdot \eta_{4} \cdot \eta_{5} \cdot \eta \\
& \eta_{1}=1-\left[(f) \cdot(\mu)\left(\frac{M_{2}}{i} \pm \frac{M_{2}}{i}\right)\right]
\end{aligned}
$$

where: $\mu=0.1, f=2.6$, And $(+)$ denotes to external missing while $(-)$ denotes to internal missing.

$$
\begin{array}{ll}
\eta_{1}=0.966 & \eta_{2}=0.975 \\
\eta_{3}=0.975 & \eta_{4}=0.975 \\
\eta_{5}=0.971 & \eta_{6}=0.995 \\
\eta=\eta_{1} \cdot \eta_{2} \cdot \eta_{3} \cdot \eta_{4} \cdot \eta_{5} \cdot \eta_{6}=0.86
\end{array}
$$

At $M 1=112.35 \mathrm{kgf} . \mathrm{mm}$;

At $\mathrm{M} 2=240.35 \mathrm{kgf} \cdot \mathrm{mm}$;

$$
M_{10}=\frac{M_{1}}{i} \eta=0.3611 \text { kgf.mm }
$$

$$
M_{20}=\frac{M_{1}}{i} \eta=0.7725 \mathrm{kgf} . \mathrm{mm}
$$

The dimension values of centrifugal brake parameters are:

$$
\begin{array}{ll}
a=4,667 \mathrm{~mm} ; & b=5,013 \mathrm{~mm} ; \\
r=8,75 \mathrm{~mm} ; & \rho=5,765 \mathrm{~mm} ; \\
d=5,833 \mathrm{~mm} . &
\end{array}
$$

Then:

$$
\begin{array}{r}
K_{1}=132057.9819 \quad \& \quad K_{2}=128686.4431 \\
\omega_{\text {ocrit min }}=\sqrt{\mathrm{K}_{1} \times \mathrm{M}_{10}} \\
=218.37 \mathrm{rad} / \mathrm{sec}=12511.768 \mathrm{deg} . / \mathrm{sec} .
\end{array}
$$




$$
\begin{aligned}
\omega_{\text {ocrit. } \min } & =\sqrt{K_{1} \times M_{1}} \\
= & 315.2939 \mathrm{rad} / \mathrm{sec}=18065.0128 \mathrm{deg} . / \mathrm{sec}
\end{aligned}
$$

By means of transmission gear ratio, these angular velocities are transmitted to the movable part,

$\omega$ - the current angular velocity of rotation of a rotating element of R.M

$\omega_{\min }$ - the minimum angular velocity of rotation of a rotating element of R.M

$\omega_{\max }$ - the maximum angular velocity of rotation of a rotating element of R.M

$$
\begin{aligned}
& \omega_{\min }=\omega_{\circ \text { crit. } \min } / \mathrm{i} \\
& =46.764 \mathrm{deg} . / \mathrm{sec} \\
& \omega_{\max }=\omega_{\circ \text { crit. } \max } / \mathrm{i} \\
& =67.520 \mathrm{deg} . / \mathrm{sec}
\end{aligned}
$$

Roughly we determine average angular velocity of rotation of a rotating element of R.M:

$$
\omega_{\mathrm{av}}=\frac{\omega_{\min }+\omega_{\max }}{2}=57.142 \mathrm{deg} . / \mathrm{sec}
$$

Average time of rotation of a solar panel array to an angle $90^{\circ}, \mathrm{T}_{\mathrm{av}}=90 / 57.142=$ $1.6 \mathrm{sec}$, which satisfies the required time $(T=1.5 \mathrm{sec})$.

\section{STRESS ANALYSIS OF ROTATION MECHANISM}

Mathematical model of rotation mechanism is constructed using finite element computer package (ANSYS). Each finite element package provides a library of structure elements, such as beams, shells, and solid elements. The RM is modeled by connecting elements together with geometry, material properties, and boundary conditions appropriate to model the design. Details of geometry and boundary conditions are discussed in the next section.

Forces and Moments obtained from solar panel analysis are given by satellite structure strength analysis engineer,

$$
\begin{aligned}
& F_{x}=-12.90775, F_{y}=-0.533 E-5, F_{z}=-16.50722 \mathrm{Kg} \cdot \mathrm{mm} / \mathrm{s}^{2} \\
& M_{x}=7280.538, M_{y}=-5794.947, M_{z}=574.5542 \mathrm{Kg} \cdot \mathrm{mm}^{2} / \mathrm{s}^{2}
\end{aligned}
$$

Strength analysis of rotation mechanism is performed to check if the RM structure will stand its loads at stoppage moment. It is recommended to run modal analysis to make sure the natural frequencies of solar panel will not match the RM ones. Material is АMГ6 and assumed to be linear isotropic. $\left(E=69 \mathrm{E}+6 \mathrm{Kg} \cdot \mathrm{mm} / \mathrm{s}^{2} / \mathrm{mm}^{2}, \sigma_{U}\right.$ $=320 \mathrm{MPa}, \sigma_{Y}=200 \mathrm{MPa}$ ). Solid45 elements are used which has 8 nodes to save model space. it is recommended to use Solid92 elements 


\subsection{Analysis Technique}

Each subassembly consists from different mechanical parts. These mechanical parts are fixed together through bolts. The analysis for these bolts is not preformed for the simplification of the model. So, these mechanical parts are fixed together.

Next step is to couple between three subsystems. Fig. 10 illustrates the RM full assembly at the stoppage time. The three subsystems rotate about one axis. So, couple 3 degree of freedom at the center in the direction of $(x, y, z)$ at each end (along the rotating axis) of subsystems, See Fig.7.

Bolt of subsystem ' $B$ ' is coupled with the internal gear of subsystem ' $C$ ' in the direction perpendicular to axis of rotation (Fig.8). Brackets of subsystems ' $A$ ', ' $B$ ' are coupled on the contact surface area between them in the direction perpendicular to that area (Fig.9).

Apply loads that were calculated from solar panel simulation (using ANAYS) on the rotation subassembly (B) as shown in Fig.10. Apply displacement boundary condition (fix all degrees of freedom) on the subassembly ' $A$ ' along the two cylindrical area through which the subassembly is fixed to satellite body (Fig. 10). Run Static analysis for strength analysis of each structure of RM. Figure11 shows
finite element model of RM.

Although the function of a mechanism is to release solar panel, it is also a structure, subject to all loading environments that apply to other satellite structure components So, we must predict loads and determine structural strength and life for all potentially critical events, satisfy stiffness requirements, and do environmental tests.

\subsection{Static stress analysis}

Stress analysis results are covered in this section while testing is preformed during the strength Mockup phase. The following analysis results will be checked, deformation of RM and Von-Misses stress for each subassembly are compared to the ultimate strength of the AMr6.

Fig. 10 shows the deformation of RM at stoppage time. Deformations are small and will not affect the structure of solar panel. It would be a better model in case of integration of solar panel with RM in one model or using the superelement (substructure) method. 


\subsubsection{Subassembly 'A' stress analysis results}

Maximum equivalent stress is $0.26 \mathrm{MPa}$ at the connection area with subassembly ' $\mathrm{B}$ ' and ' $C$ '. Subassembly structure ' $A$ ' is safe (Fig.12).

\subsubsection{Subassembly ' $B$ ' stress analysis results}

Maximum equivalent stress is at the bolt. Its location is at area where is in contact with internal Gear of subassembly ' $C$ '. The structure is over safe. (Fig. 13). $\sigma_{\mathrm{Max}}=0.106 \mathrm{MPa}$.

\subsubsection{Subassembly ' $C$ ' stress analysis results}

Maximum equivalent stress at location of contact of Bolt of subassembly ' $B$ ' $(0.31$ $\mathrm{MPa}$ ), so structure of subassembly ' $\mathrm{C}$ ' is safe. Another location to be checked is the area of contact between the two brackets of subassemblies. (Fig.14).

Due to the brake regulator that makes the opening of the RM with low rotational acceleration, the structure of the RM experiences low stresses. Static strength analysis of RM proves that it is safe.

\section{ROTATION MECHANISM RELIABILTY ANALYSIS}

Rotation mechanism reliability can be divided to three parts; function of rotation of solar panel, function of fixation of solar panel, and function of in destruction of rotation mechanism. Assuming independence of the process of the rotation mechanism functioning, probability of no-failure of the rotation mechanism is determined from,

$$
\begin{aligned}
& P_{C}=\prod_{i=1}^{3} P i \\
& P_{c} \quad \text { Probability of no-failure of the RM. } \\
& P_{1} \quad \text { Probability of the rotation of the solar panel. } \\
& P_{2} \text { Probability of the fixation of the solar panel. } \\
& P_{3} \quad \text { Probability of in destruction of the rotation mechanism. }
\end{aligned}
$$

P3 is assumed to equal to ' 1 ', as during design phase safety factors (due to randomness of acting loads and material characteristics) were applied, and rotation mechanism went through strength tests during the QR (qualification phase). P2 is also assumed to equal to ' 1 ', as the process of fixation is adjusted manually at the rotation mechanism development phase. P1 is determined at the condition of the worst case (maximum load). 
Assume that the spring force and the load are distributed according to the normal low. In this case, probability of an event considering the fact that the spring force will be greater than the applied load,

$$
\begin{aligned}
& P_{C}=\Phi\left[\frac{Y-N}{\sqrt{\delta_{y}^{2}+\delta_{N}^{2}}}\right] \\
& \Phi \text { normal distribution. } \\
& \mathrm{Y} \text { average value of the spring force (moment). } \\
& \mathrm{N} \text { average value of applied load on spring. } \\
& \delta_{\mathrm{y}} \text { mean square deviation of the spring force. } \\
& \delta_{\mathrm{N}} \text { mean square deviation of the applied load. }
\end{aligned}
$$

From previous experience the following is assumed,

$$
\delta_{y}=3.3, \quad \delta_{N 1}=0.0034, \quad \delta_{N 2}=4.7
$$

Average value of spring moment,$Y=100 \mathrm{Kgf} . \mathrm{mm}$. $\delta_{\mathrm{N} 1}$ is mean square deviation of the moment of resisting force that results from the solar panel relative to the rotation axis $\left(\mathrm{N} 1=\mathrm{M}_{\text {Inertia }}=0.0675 \mathrm{Kgf} . \mathrm{mm}\right)$, while $\delta_{\mathrm{N} 2}$ is the moment of resisting force of cables bend relative to the rotation axis ( $2=70 \mathrm{Kgf} . \mathrm{mm}$ ).

$$
\begin{aligned}
P_{C} & =\Phi\left[\frac{100-70-0.0675}{\sqrt{3.3^{2}+4.7^{2}+0.0034^{2}}}\right] \\
& =\Phi[5.212]=0.9999999
\end{aligned}
$$

Reliability analysis of rotation mechanism was determined based on classic probability theory. Better reliability analysis is to be calculated applying stochastic finite element analysis [10], which is a new emerging field of study. Stochastic finite element analysis integrates the reliability methods and the deterministic finite element method to estimate risk of any structure. It replaces the sensitivity analysis, which involves extensive running times.

\section{CONCLUSION}

After orbit insertion of satellite, solar panel deployment is a tense phase. The main issue here is to recognize this risk from the start and adopt good design practices and setting high standards during all project phases (manufacturing, assembly and testing). One goal of this paper is to familiarize satellite designers with a number of selected issues related to space applicable rotation mechanism. Design of both the spring and centrifugal brake are presented. Modeling of rotation mechanism using 
finite element package (ANSYS) was presented and its strength was determined at the most critical point of its function (stoppage moment). Rotation mechanism reliability analysis is discussed. Reliability-based design of rotation mechanism is proposed to be the next point of research. Stochastic finite element analysis is one of the new emerged research topics, and due to the simplicity of rotation mechanism finite element modeling, it can be applied in the Reliability-based design process.

\section{REFERENCES}

[1] Central Machine Tool Institute - Bangalore, "Machine Tool Design Handbook", McGraw-Hill, Inc. (1982).

[2] NARSS report, "Satellite Preliminary Design. Composition and Main Characteristics" (2003)

[3] NARSS report, "Satellite Structure. Verification plan" (2003).

[4] Thomas P. Sarafin," Spacecraft Structures and Mechanisms. From Concept to Launch". Kluwer Academic Publishers (1998).

[5] Joseph Shigley," Mechanical Engineering Design “. McGraw-Hill, Inc (2003).

[6] Peter L. Conley, "Space Vehicle Mechanisms, elements of Successful Design". JOHN WILLEY \& SONS, Inc (1998).

[7] Russell C. Hibbeler, "Structural Analysis,3rd". PRENTICE HALL, Inc (1995).

[8] Nelson R. Bauld, "Mechanics of Materials". PWS Publishers (1986).

[9] Tomas G. Beckwith, "Mechanical Measurements, $5^{\text {th" }}$. Addison-Wesley Publishing Company (1993).

[10] Achintya Haldar and Sandkaran Mahadevan, "Reliability Assessment Using Stochastic Finite Element Analysis". JOHN WILLEY \& SONS, Inc (2000).

[11] GOST Standards; OST 92 - $8847-77$.

[12] Ty 3 - $1002-77$; technical specification of YOZHNOYE design office.

[13] GOST Standards; OCT 3-2561-74.

[14] GOST Standards; OST $92-8847-77$. 


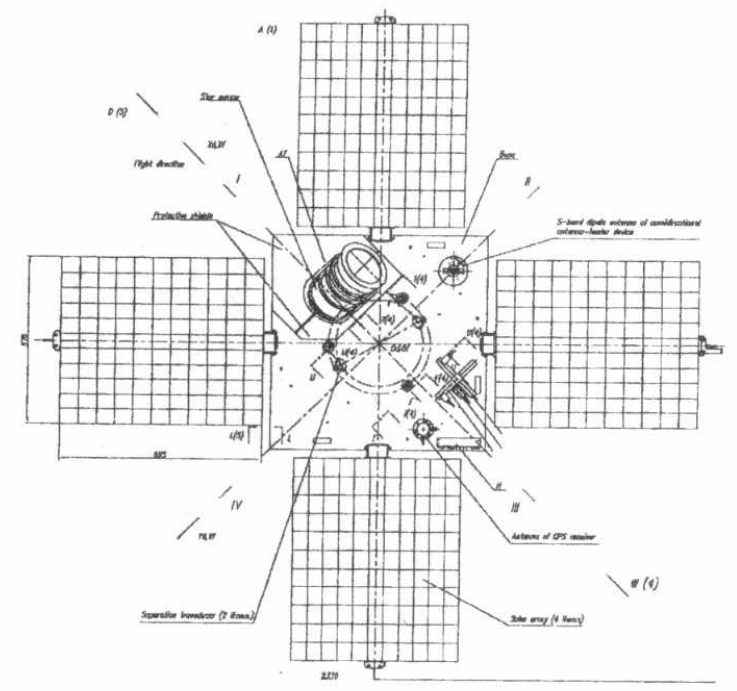

Fig.1. Top View of Micro-Satellite Structure.

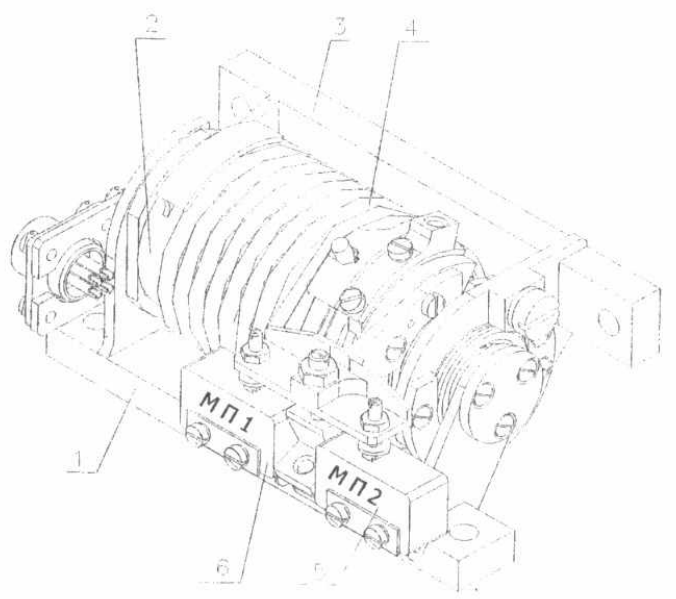

1 - housing,

2 - centrifugal governor,

3 - housing,

4 - spring,

5,6-microswitches.

Fig.2. Overall view of rotation mechanism 

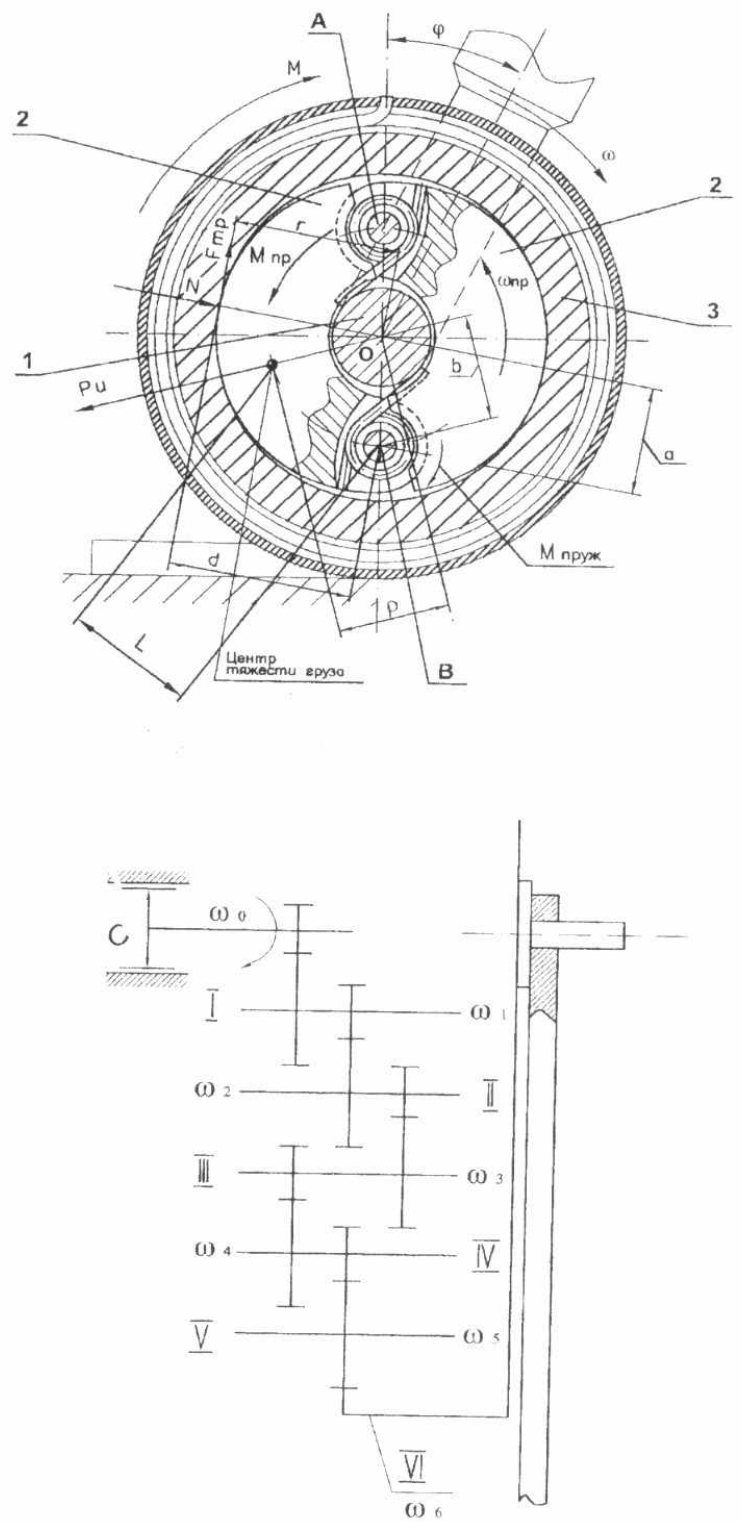

Fig.3. Centrifugal Brake of Rotation Mechanism 


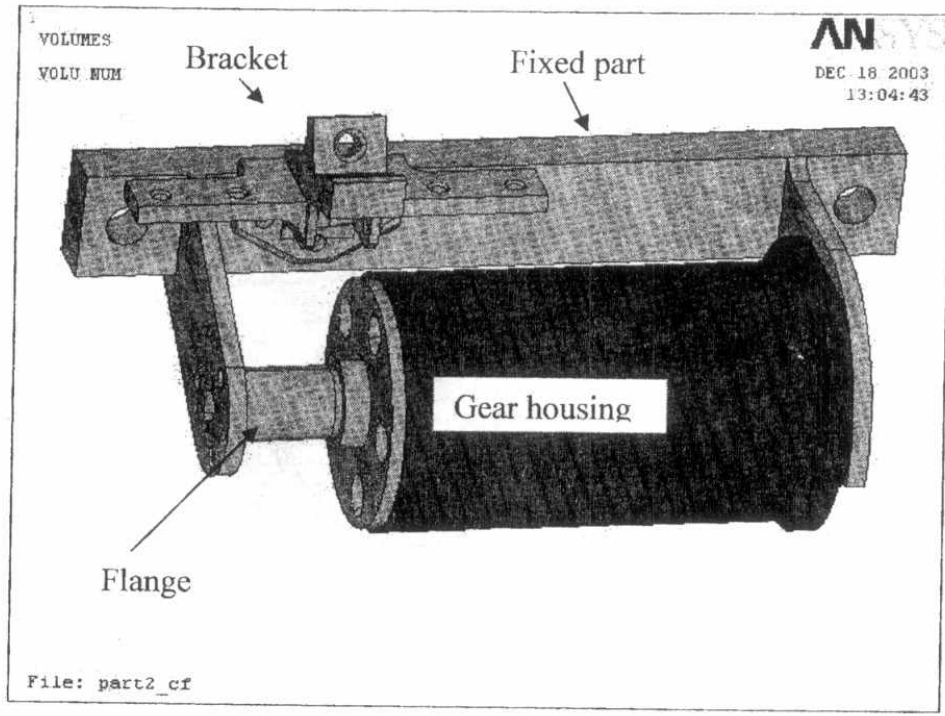

Fig. 4. Subassembly 'A', Fixed to Base Plate.

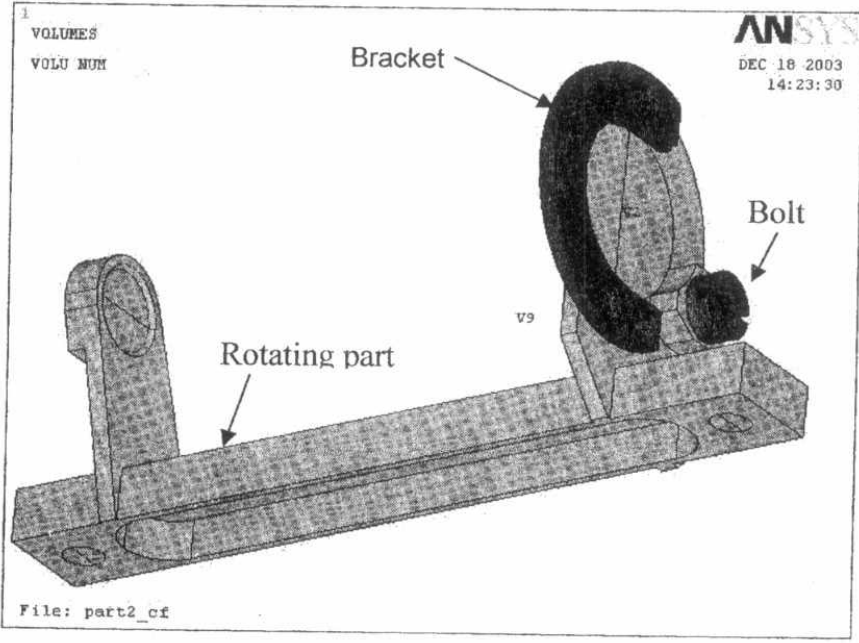

Fig. 5. Subassembly 'B', Fixed to Solar Panel. 


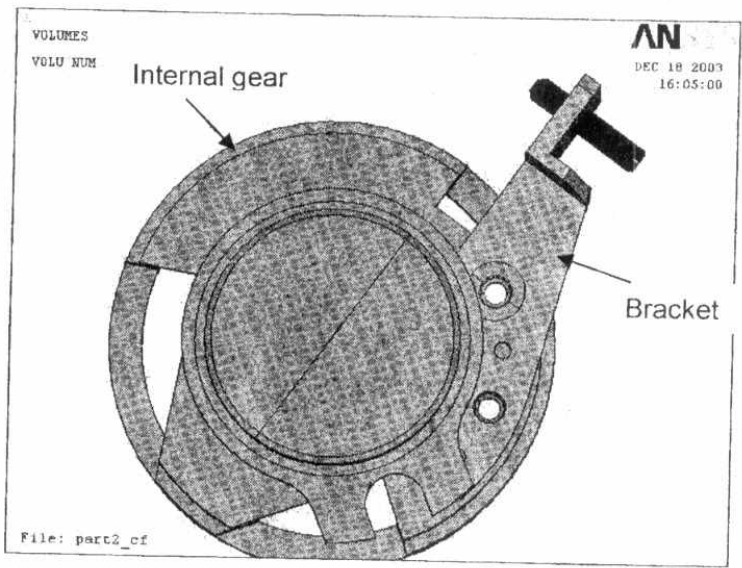

Fig. 6. Subassembly 'C'.

Couple these nodes for

$\mathrm{X}, \mathrm{Y}, \mathrm{Z}$, in one coupling

set.

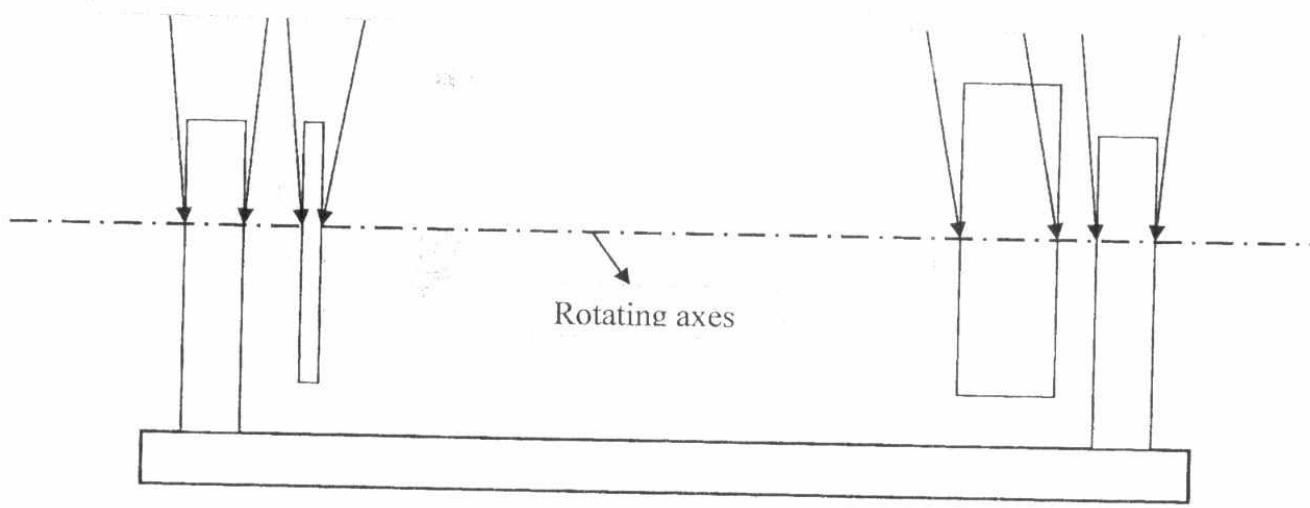

Couple these nodes for $\mathrm{X}, \mathrm{Y}, \mathrm{Z}$, in one coupling set.

Fig. 7. Coupling Scheme Between RM 'Subsystems. 


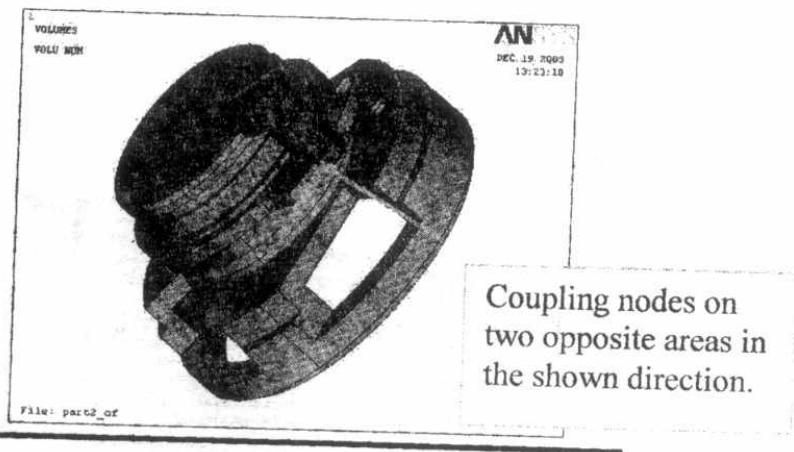

Fig. 8. Coupling between Internal Gear and Bolt.

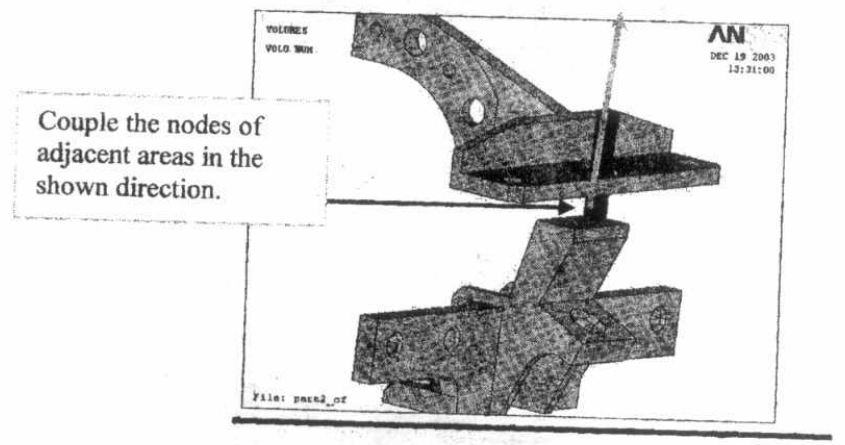

Fig. 9. Coupling between Two Brackets.

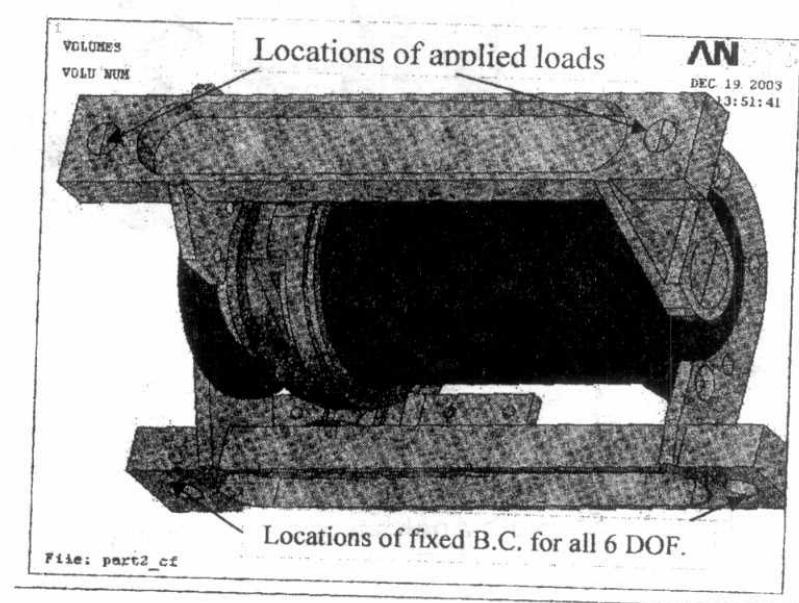

Fig. 10. Boundary Conditions and Applied Loads. 


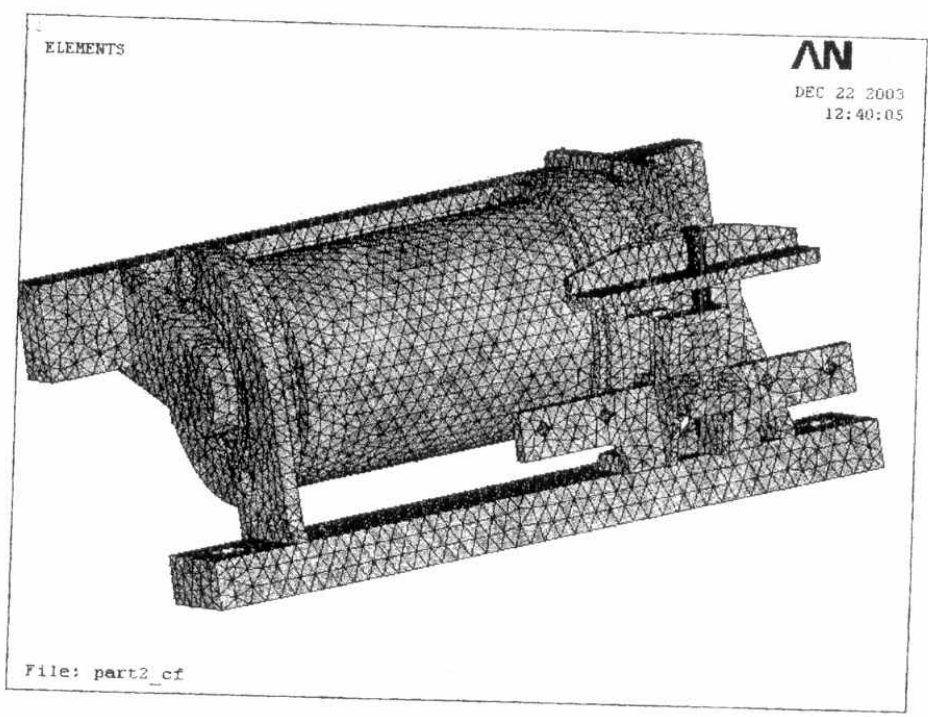

Fig. 11. Meshed Rotation Mechanism.

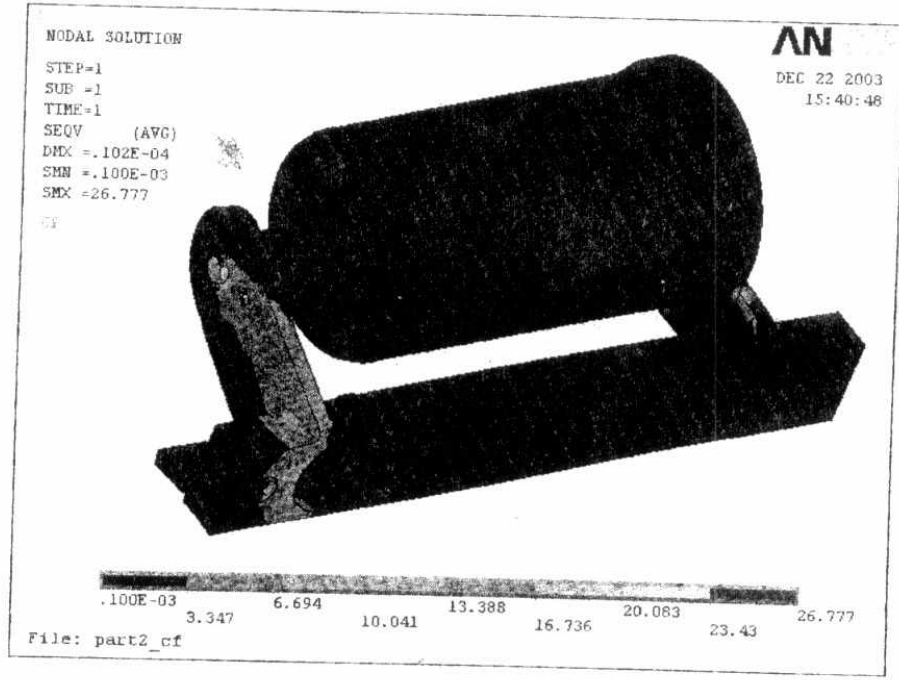

Fig. 12. Strength Analysis of Subassembly 'A'. 


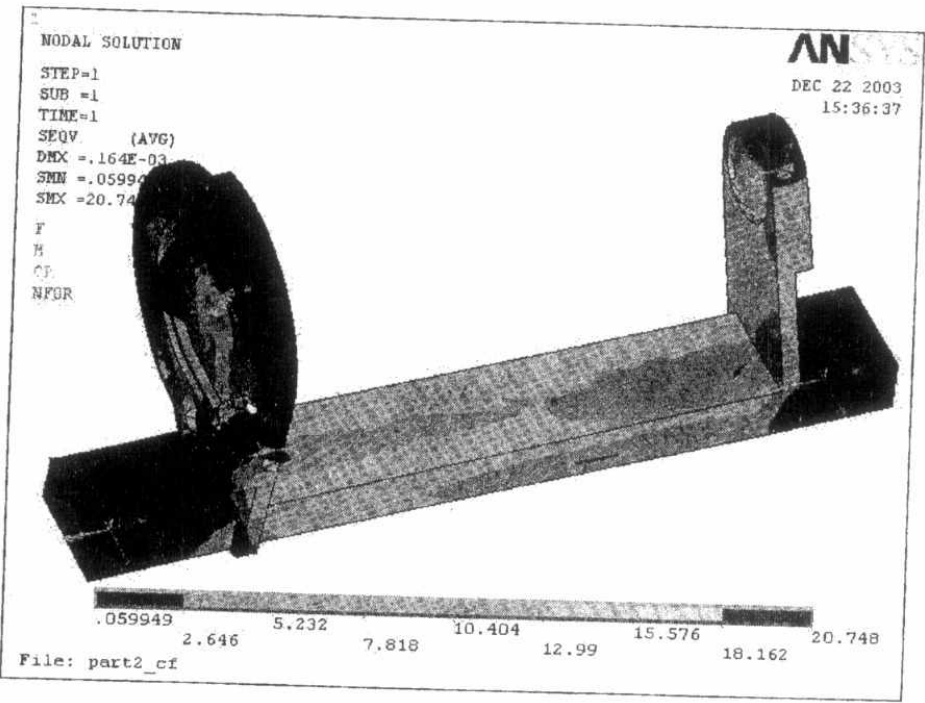

Fig. 13. Strength Analysis of Subassembly 'B'.

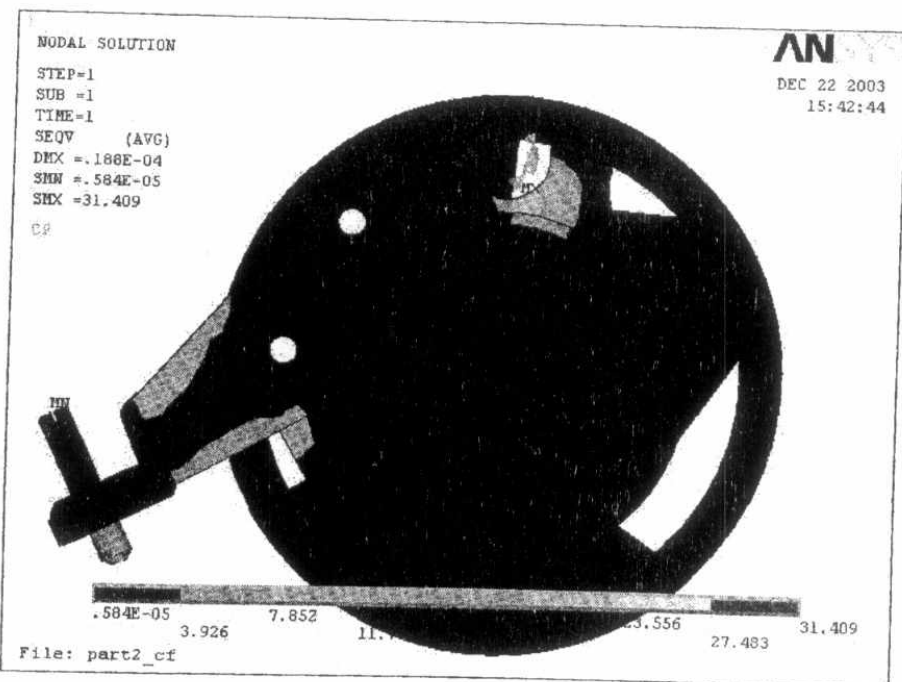

Fig. 14. Strength Analysis of Subassembly ' $C$ '. 